\title{
To Cryo or Not to Cryo? A Consideration of Length Scales During Macromolecule Sample Preparation
}

Kelly Parker ${ }^{1}$, Justin Modica ${ }^{2}$, Charlene Wilke ${ }^{3}$, Roberto dos Reis ${ }^{4}$, Milan Mrksich $^{2}$ and Vinayak Dravid ${ }^{4}$ ${ }^{1}$ Department of Materials Science and Engineering, Northwestern University, Evanston, Illinois, United States, ${ }^{2}$ Department of Biomedical Engineering, Northwestern University, United States, ${ }^{3}$ The NUANCE Center, Northwestern University, United States, ${ }^{4}$ Department of Materials Science and Engineering, Northwestern University, United States

Advances in cryo-transmission electron microscopy (TEM), particularly hardware such as direct electron detectors and software such as reconstruction algorithms and sparse collection methods, have begun to address the challenges inherent in biological imaging: low contrast and susceptibility to beam damage [1]. However, Cryo-EM is not without its drawbacks; it is low-throughput compared to other imaging methods, prone to radiolytic damage from inelastic scattering, and requires potentially prohibitive expertise and equipment. Proteins and protein assemblies represent a variety of length scales, and the construct size and imaging requirements may make room temperature preparation a viable alternative. This paper will examine the decision "to cryo or not to cryo", particularly for imaging the architecture of assembled proteins. We will describe the decision flow based on a number of factors, exemplified through a protein construct known as a "megamolecule" that undergoes a conformational change when exposed to an external stimulus [2].

The flowchart in Figure 1 provides our considerations for sample preparation. When we receive a protein assembly, precedent encourages cryo-imaging; however, negative staining may be a preferable method. Our first consideration is the requisite resolution to distinguish features. If this resolution is smaller than the threshold for typical microscopes at room temperature, cryo-imaging will likely be necessary. This resolution limit is approximately $1 \mathrm{~nm}$ in our case, as negative stains reduce resolution according to their grain size [3]. If this decision directs towards cryo-EM, we must also consider whether the sample is stable under cryo-conditions; the electron dose needed to achieve sufficient signal-to-noise and the prevalence of radiolytic damage in cryo-EM might require extended techniques to reduce dose [4].

When cryo-resolution is not required, negative staining should be considered because it is comparably high-throughput, reduces dose due to higher contrast, and is more accessible with a variety of equipment. However, decisions must also occur based on several other factors. Though negative stains rapidly fix structures, the process of staining and drying (often at low $\mathrm{pH}$ ) can cause protein breakup and aggregation. The gentler cryo-preparation is appropriate if homogenous populations are required and fragile samples are destroyed during the staining process. Cryo-EM should similarly be used for single particle analysis as the affinity of proteins for negatively-charged films will lead to a preferred orientation [5]. The use of extended techniques such as spectroscopy or micro-electron diffraction (micro-ED), among others, can also warrant cryo-preparation, as stains potentially interfere with signal and resolution. Finally, cryo-EM is a lower-throughput method, particularly when compared with scanning TEM (STEM) in SEM [6]; when the screening of many samples is required, this is better-done using room temperature imaging.

This decision flow is exemplified through the images in Figure 2. The sample of interest, a megamolecule protein construct, is shown in the insets; the protein scaffolds undergo intramolecular reaction through enzyme domains to convert linear molecules into macrocycles [7-8]. Our imaging effort should both show examples of the stimulus-dependent conformations and yield a statistical analysis of construct size. This 
megamolecule is formed from well-understood proteins, so imaging must resolve the individual 3-4 nm proteins and observe their orientation in the construct. The required resolution is larger than our $1 \mathrm{~nm}$ threshold, not necessitating cryo-imaging. The megamolecules were stained with $0.75 \%$ uranyl formate, and the TEM images in Figure $2 a-b$ were used to distinguish between the cyclized and linear structures. However, individual proteins and aggregates were also observed. This non-homogenous population could not be used to analyze the size difference between conformations; proteins experienced breakage and shrinking during preparation, so cryo-EM (Figure 2c) was necessary to measure the size of the constructs. In this case, the resolution did not necessitate cryo-EM, but additional imaging requirements and the fragility of the sample resulted in a shift from room temperature to cryo-imaging. Further cryo-EM and class averaging of the cyclized and linear structures will be used to analyze their size difference and the relative percentage of each population.

While cryo-EM is the typical methodology for macromolecule imaging, there are many cases where room temperature preparation provides the necessary results more quickly and accessibly. We describe our decision flow applied to the imaging of a stimulus-responsive protein megamolecule. While we specifically compared cryo-EM and room-temperature negative staining, many other preparation and imaging techniques are effective; these include cryo-focused ion beam (FIB) sectioning, high-pressure freezing, and high-throughput STEM imaging in SEM. Sample preparation ultimately requires iteration, but making preemptive decisions based on the necessary resolution and imaging goals can simplify and expedite the preparation process.

Acknowledgments: This work is supported by the Army Research Office (W911NF1810200), the National Science Foundation Graduate Research Fellowship (DGE-1842165), and the Ryan Fellowship, and partially supported by the Air Force Office of Scientific Research (FA9550-12-1-0280). This work made use of the Electron Probe Instrumentation Center and BioCryo facilities of Northwestern University's NUANCE Center, which has received support from the Soft and Hybrid Nanotechnology Experimental (SHyNE) Resource (NSF ECCS-1542205); the MRSEC program (NSF DMR-1720139) at the Materials Research Center; the International Institute for Nanotechnology (IIN); the Keck Foundation; and the State of Illinois, through the IIN. 


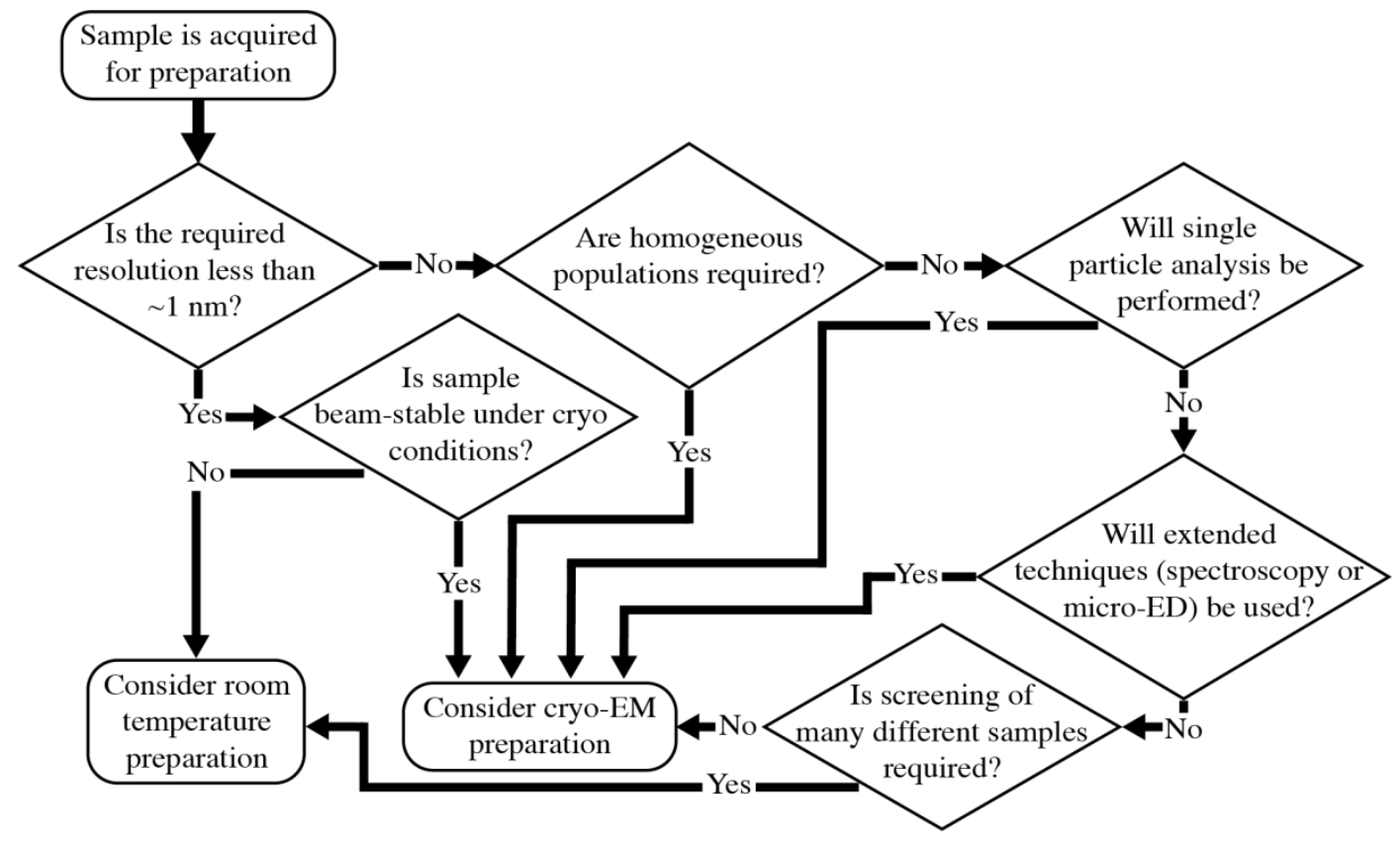

Figure 1. Figure 1. Flowchart describing conditions that lead to either cryo-EM or room temperature negative staining for macromolecular constructs.
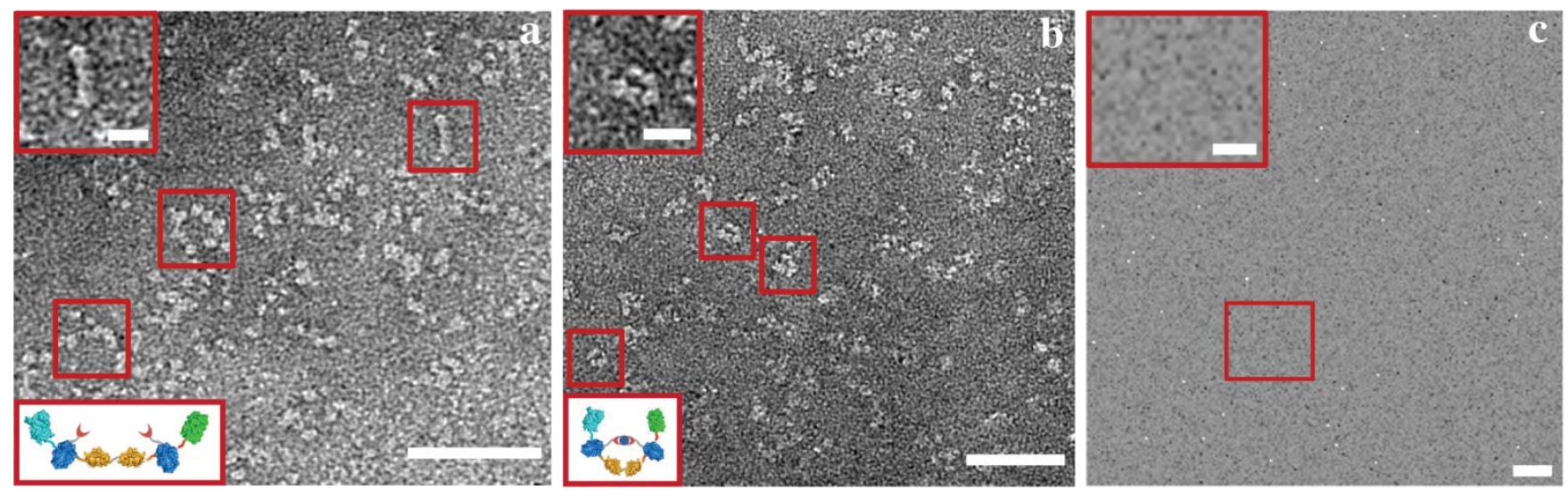

Figure 2. Figure 2. a-b) Negative stained TEM images at $100 \mathrm{kV}$ showing a) the linear megamolecule conformation and b) the cyclized conformation. Upper insets show a magnified structure, and lower insets schematically depict the expected conformation. c) Cryo-TEM image at $300 \mathrm{kV}$ showing a partially cyclized structure. Sharp white and dark spots originate from detector noise after filtering. Scale bars: 50 $\mathrm{nm}$ and $10 \mathrm{~nm}$ (inset).

\section{References}

[1] Koning R. I., Koster A. J., Sharp T. H. Annals of Anatomy 217 (2018), p. 82-96.

[2] Modica, J. A., Lin, Y., Mrksich, M. J. Am. Chem. Soc. 140 (2018), p. 6391-6399.

[3] Gallagher J. R., Kim A. J., Gulati N. M., Harris A. K. Current Protocols in Microbiology 54, 1 (2019).

[4] Karuppasamy M., Nejadasl F. K., Ravelli R. B. G. et al. J. Synchrotron Rad. 18 (2011), p. 398-412.

[5] Meyerson J. R., Rao P., Subramaniam S. et al. Scientific Reports 4, 7084 (2014).

[6] Parker, K., Zhou, S., Mrksich M., Dravid, V. et al. Microscopy and Microanalysis 26, S2 (2020), p. 1026-1028. 
[7] Taylor E. L., Metcalf, K. J., Mrksich, M., Goodson, T. et al. J. Am. Chem. Soc. 140 (2018), p. 1573115743.

[8] Metcalf K. J., Kimmel B. R., Parker K. A., Dravid V. P., Mrksich M. et al. Bioconjugate Chem. 32 (2021), p. 143-152. 